\title{
Field dependence of state populations in pump-probe pulses
}

\author{
Xingqiang $\mathbf{L U}^{\mathrm{c}}$, Wei Guo ${ }^{\mathrm{a}, \mathrm{b} *}$, \\ a. School of Electrical Engineering, University of South China, Hengyang 421001, China \\ b. State Key Laboratory of Molecular Reaction Dynamics, Dalian Institute of Chemical Physics, Dalian \\ 116023, China \\ c. School of Nuclear Science and Technology, University of South China, Hengyang 421001, China
}

\section{ABSTRACT}

The effects of pulse width, laser wavelength and laser intensity on the state populations of NaI molecule driven by a pump-probe pulse were studied via employing the time-dependent wave packet approach. Wave packet moves periodically with roundtrip time $1000 \mathrm{fs}$. That the wave packet bifurcates in the crossing region affects state populations. The excitation probability increases and dissociation probability decreases with increasing pulse width. The excitation probability approaches the maximum at the resonance pump wavelength $318 \mathrm{~nm}$, and the dissociation probability decreases with increasing pump wavelength. The excitation probability increases with increasing pump intensity while the dissociation probability does not vary. The probe wavelength and probe intensity have no effect on state populations. The wave packet motion and selective distribution of state populations, excitation and dissociation of molecule can be achieved by adjusting laser parameters. The results can provide some important basis for realizing quantum manipulation of molecules experimentally.

Keywords:

Intense femtosecond pump-probe pulse; Nonadiabatic effect; Time-dependent wave packet approach; Population transfer; Dissociation probability

\footnotetext{
* Corresponding author. Tel: +86 734828 2373; fax: +86 7348282733.

E-mail address: vella99@163.com (W. Guo)
} 


\section{Introduction}

With the development of ultrashort and ultrastrong laser pulse technology, more and more researches have focused on the control of molecular dynamics in real time. Controlling the evolution of wave packet will be a benefit for light manipulation of molecular processes experimentally. State populations map the wave packet dynamic information of the electronic state, are found to be sensitive to the parameters of the laser fields, and have been studied in multi-level molecular systems [1-14].

$\mathrm{NaI}$ molecule is a typical molecule with a avoided crossing between two nonadiabatically coupled electronic states at internuclear distance $7 \AA$, and has been studied experimentally and theoretically. Braun et al. [1] and Charron and Suzor-Weiner [2] presented that the wave packet moves periodically on the electronic state potentials of $\mathrm{NaI}$ molecule, which induces the periodical variation of photoelectron spectra. Yao et al. $[3,4]$ suggested that the first dissociation probability decreases with increasing pump wavelength. The photoelectron spectra are dependent on the delay time. Arasaki et al. [5-7] indicated that the total ion signal oscillates periodically with increasing delay time. Miao et al. [8,9] and Liu et al. [10] suggested that the delay time affects state populations and photoelectron spectra. Ma et al. [11] presented that the pump wavelength affects state populations. Zhao et al. [12] studied the influence of field-free orientation on the predissociation dynamics of the NaI molecule. Xiong et al. [13] presented that the wavelength affects state populations of CsI molecule, the populations of excited state approaches the maximum with the resonance excitation wavelength. Zhu et al. [14] suggested the delay time affects state populations of CsI molecule.

The studies on the nonadiabatic effects on state populations of $\mathrm{NaI}$ molecule above mostly focused on the influence of pump-probe delay time. The effects of the pulse width and laser intensity on state populations of $\mathrm{NaI}$ molecule have not been reported. This paper presents new data on the influence of pulse width, laser wavelength and intensity on state populations of $\mathrm{NaI}$ molecule driven by pump-probe pulses via time-dependence quantum wave packet method.

\section{Computation details}

Three states (the ground state $X$, the excited state $A$ and the ion ground state $I$ ) are involved in the multiphoton ionization of $\mathrm{NaI}$ molecule [1-3], as shown in Fig.1. The transition from $X$ to $A$ is 
induced by resonant one-photon absorption, then the excited molecule is ionized through single-photon absorption after the delay time, and the emitted photoelectron is detected from $I$.

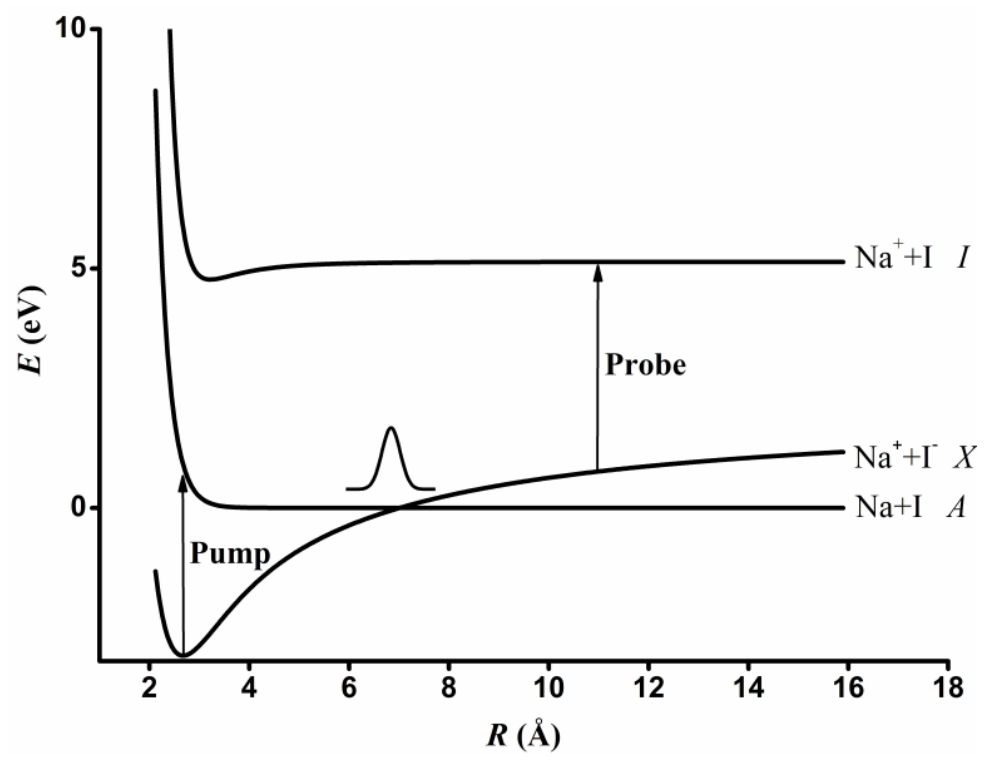

Fig. 1. Potential energy curves of NaI molecule.

The wave function for the three-state model can be written as

$\boldsymbol{\Psi}=\left(\Psi_{X}, \Psi_{A}, \Psi_{I}\right)^{T}$,

Where $\Psi_{X}, \Psi_{A}$ and $\Psi_{I}$ are the wave functions for the states $X, A$, and $I$, respectively. The ion ground state is a continuum state and can be discretized into a band of quasicontinuum states. The $\Psi_{I}$ can be further expressed as

$\Psi_{I}=\left(\psi_{1}, \psi_{2}, \ldots, \psi_{N}\right)^{T}$

where $N$ represents the number of discrete states of $\mathrm{NaI}$ ion. The wavefunctions $\Psi_{I}$, within the Born-Oppenheimer approximation can be obtained by solving the time-dependent Schrödinger equation

$i \hbar \frac{\partial}{\partial t}\left(\begin{array}{c}\Psi_{X} \\ \Psi_{A} \\ \psi_{1} \\ \psi_{2} \\ \vdots \\ \psi_{N}\end{array}\right)=-\frac{\hbar^{2}}{2 \mu} \frac{\partial^{2}}{\partial R^{2}}\left(\begin{array}{c}\Psi_{X} \\ \Psi_{A} \\ \psi_{1} \\ \psi_{2} \\ \vdots \\ \psi_{N}\end{array}\right)+\boldsymbol{V}(R, t)\left(\begin{array}{c}\Psi_{X} \\ \Psi_{A} \\ \psi_{1} \\ \psi_{2} \\ \vdots \\ \psi_{N}\end{array}\right)$,

where $\mu$ is the reduced mass and $R$ is the internuclear distance, while the potential matrix 
$\boldsymbol{V}(R, \mathrm{t})$ can be explicitly written as

$$
\boldsymbol{V}(R, \mathrm{t})=\left(\begin{array}{cccccc}
V_{X X} & W_{X A} & 0 & 0 & \ldots & 0 \\
W_{A X} & V_{A A} & W_{A I} & \ldots & \ldots & W_{A I} \\
0 & W_{I A} & V_{I I}+E_{I, 1} & 0 & \ldots & 0 \\
\vdots & \vdots & 0 & \ddots & \ldots & \vdots \\
\vdots & \vdots & \vdots & \vdots & \ddots & \vdots \\
0 & W_{I A} & 0 & 0 & \cdots & V_{I I}+E_{I, N}
\end{array}\right),
$$

where $V_{X X}, V_{A A}$, and $V_{I I}$ refer to the potential matrix elements of states $X, A$, and $I$ in the absence of laser fields. $E_{I, k}=(k-1) \Delta E_{I}(k=1,2, \ldots, N)$ is the emitted photoelectron energy. Other nonzero off-diagonal matrix elements are given by

$$
\begin{aligned}
& W_{X A}=W_{A X}=\mu_{X A}\left\{\varepsilon_{1} f_{1}(t) \cos \omega_{1} t+\varepsilon_{2} f_{2}(t) \cos \omega_{2}(t-\Delta t)\right\}, \\
& W_{A I}=W_{I A}=\mu_{A I}\left\{\varepsilon_{1} f_{1}(t) \cos \omega_{1} t+\varepsilon_{2} f_{2}(t) \cos \omega_{2}(t-\Delta t)\right\},
\end{aligned}
$$

where $\mu_{X A}$ and $\mu_{A I}$ are the transition dipole matrix elements, $\varepsilon_{1}$ and $\varepsilon_{2}$ are the amplitudes of the pump and probe pulses, $\omega_{1}$ and $\omega_{2}$ are the angular frequencies. The envelopes of the pulses $f_{1}(t)$ and $f_{2}(t)$ take Gaussian form

$$
\begin{aligned}
& f_{1}(t)=\exp \left[-4 \ln 2(t / \tau)^{2}\right], \\
& f_{2}(t)=\exp \left[-4 \ln 2((t-\Delta t) / \tau)^{2}\right],
\end{aligned}
$$

$\tau$ is the full width at half maximum (FWHM) of pulse and $\Delta t$ is the delay time between the pump and probe pulses.

The time-dependent Schrödinger equation is solved by split-operator Fourier methods rigorously $[15,16]$, once the wave function $\Psi_{i}(R, t)$ is determined, the population on each electronic state can be written as $[2,3,5,8-10,12,13]$

$$
P_{i}(t)=\int\left|\Psi_{i}(R, t)\right|^{2} d R,(i=X, A, I),
$$

The photoelectron spectrum can be obtained via $[1,2,4-6,10]$

$$
P\left(E_{I, k}\right)=\lim _{t \rightarrow \infty} \int d R\left|\psi_{k}\left(R, t, E_{I, k}\right)\right|^{2},(k=1,2, \ldots, N),
$$

In the calculation, $E_{I, k}$ span over 0-1.2 eV and $N$ equals 120. 


\section{Results and Discussion}

The effect of the delay time on the wave packet motion is considered. Figure 2 shows the evolution of wave packet on the ground state and the excited state. The wave packet moves periodically with oscillation period 1000 fs. The wave packet moves on the excited state potential within 0-200 fs, reaches the crossing point ( $R=7 \AA$ ) for the first time at $200 \mathrm{fs}$ and bifurcates: most of the wave packet transfers to the ground state [Fig. 2(a)], only a small part still moves toward large internuclear distance on the excited state [Fig. 2(b)], which eventually dissociates into $\mathrm{Na}$ and I atoms. The wave packet on the ground state is reflected at the outer turning point ( $R=11 \AA$ ) at $500 \mathrm{fs}$, returns to the crossing point at $800 \mathrm{fs}$ and splits again, where most of the wave packet transfers to the excited state and a small component remains on the ground state. The similar periodical motion and bifurcation of wave packet have been referred to $[1-8,10-12]$. The wave packet propagates at different velocity on the ground state and on excited state after the second bifurcation, and returns to the crossing point at $1100 \mathrm{fs}$ and $1200 \mathrm{fs}$, respectively, which results that the wave packet is composed of two envelopes, and is no longer a good-localization envelope, i.e. the nonadiabatic effect of NaI molecule becomes more and more obvious with increasing delay time. The wave packet on the ground state returns to the crossing point earlier than that on the excited state is referred to in Refs. $[3,4,6]$.
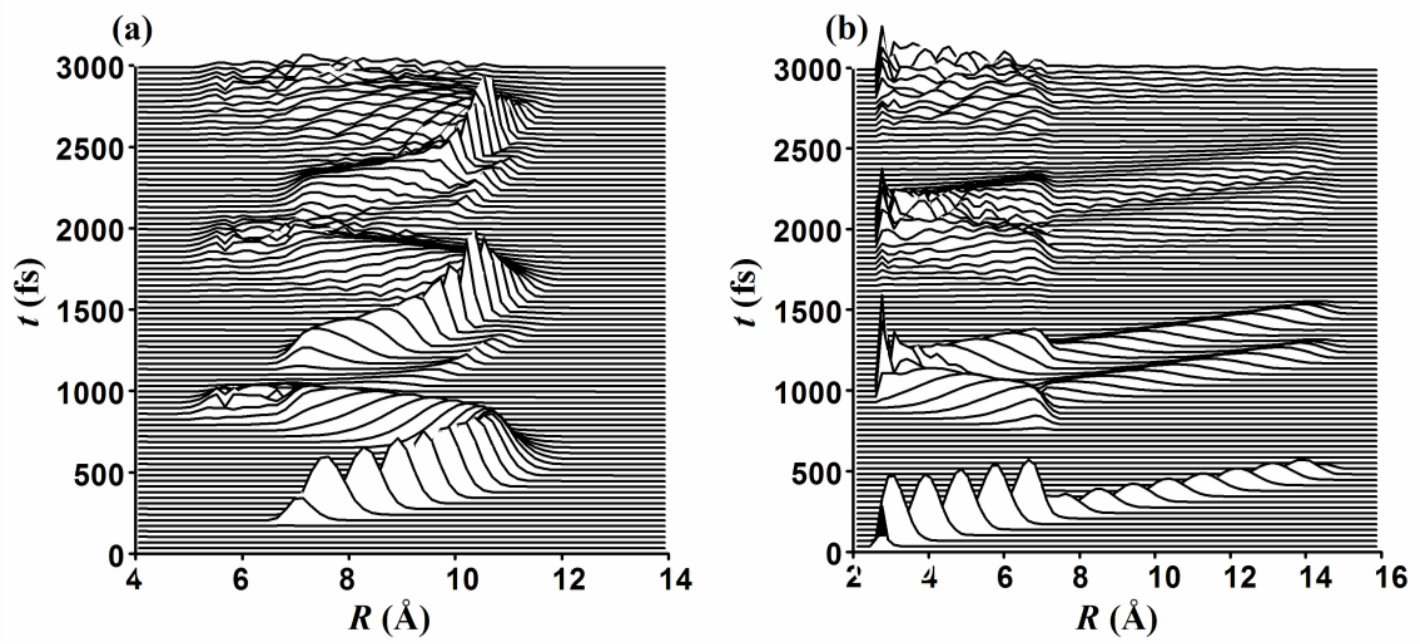

Fig. 2. The evolution of wave packet on (a) ground state $\mathrm{X}$ and (b) excited state A.

The effect of the pulse width on state populations is considered. Figure 3 demonstrates the evolution of state populations at different pulse width. All molecules are on the ground state initially until the pump laser excites them to the excited state, which makes the population of 
ground state declines [Fig. 3(a)], the population of excited state increases [Fig. 3(b)], and the sum population of three states is 1.0 [Fig. 3(c)]. The increase of the population on excited state increases (i.e. the excitation probability increases) with increasing pulse width. At $200 \mathrm{fs}$ the wave packet of excited state reaches the crossing point ( $R=7 \AA$ ) firstly and bifurcates: most of the wave packet transfers to the ground state with transfer probability $P_{X}$, while a small part remains on the excited state with transfer probability $P_{A}$, therefore the population of the ground state increases [Fig. 3(a)] and the population of the excited state declines [Fig. 3(b)], the sum of three states remains 1.0. [Fig. 3(c)]. The similar wave packet splitting induced by the nonadiabatic effects has been referred to [1-3,5-7,9-12]. Xiong et al. [13] and Zhu et al. [14] suggested that most of the wave packet of the excited state crosses to the ground state after the first bifurcation of CsI molecule. Figure 4 shows the first dissociation probability $P_{A} /\left(P_{A}+P_{X}\right) v s$. pulse width. The dissociation probability decreases with increasing pulse width. The dissociation probability is related to the wave packet propagation velocity [3]. Due that the pulse width is inversely proportional to the range of energy, the propagation velocity of the molecule on the excited state decreases with increasing pulse width, which results in increasing time to reach the crossing region, leading to decreasing dissociation probability. At 500 fs the small part of the wave packet on the excited state dissociates, therefore the population of excited state declines to 0.0, and the sum of the three states declines [Fig. 3(c)]. The decrease of the sum of the three states increases with increasing pulse width. At $800 \mathrm{fs}$, the wave packet of ground state undergoes a second bifurcation as it moves inwards, most of the wave packet crosses to the excited state, only a small part wave packet is still on the ground state, therefore the population of ground state decreases [Fig. 3(a)] while the population of excited state increases [Fig. 3(b)]. At $1200 \mathrm{fs}$, the wave packet of excited state reaches the crossing point thirdly, similar to the process at $200 \mathrm{fs}$, most of the wave packet propagates on the ground state, thus the population of ground state increases while the population of excited state decreases. At $1500 \mathrm{fs}$, the population of excited state drapes to 0.0 , similar to the process at $500 \mathrm{fs}$, the small part of wave packet on the excited state eventually dissociates, and the sum of three state declines again. The effect of the pulse width on the state populations and dissociation probability of $\mathrm{NaI}$ molecule have not studied before. 

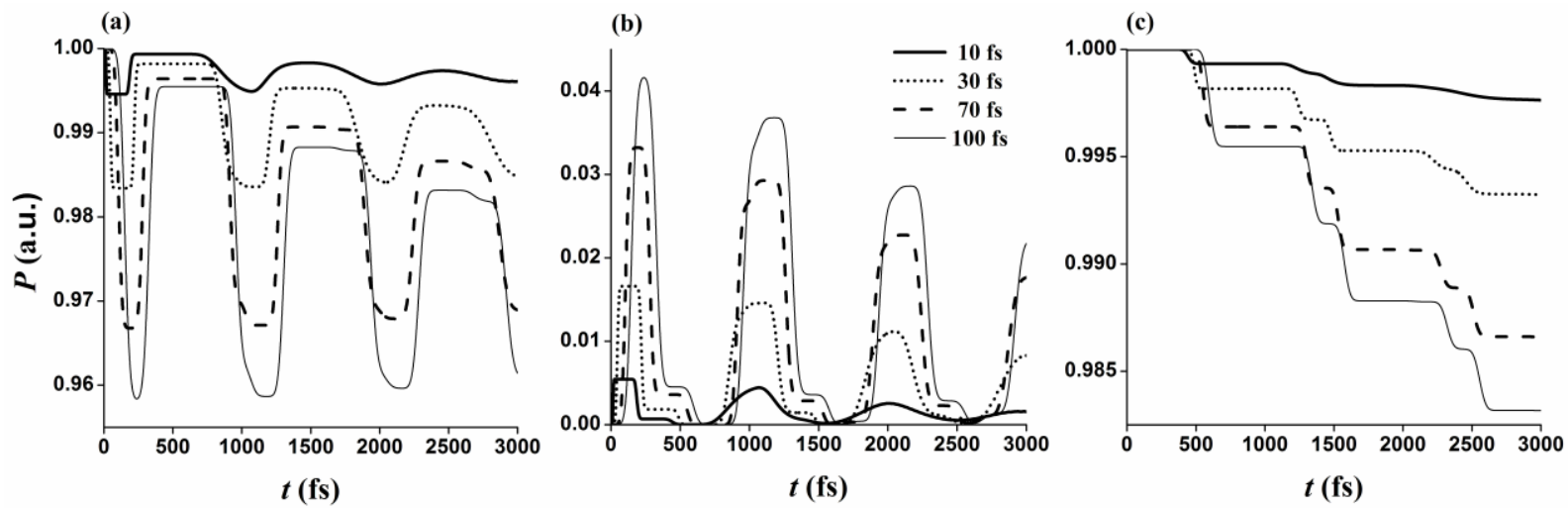

Fig. 3. The evolution of state populations at different pulse widths. (a), (b) and (c) denote ground state, excited state and the sum of the three states, respectively.

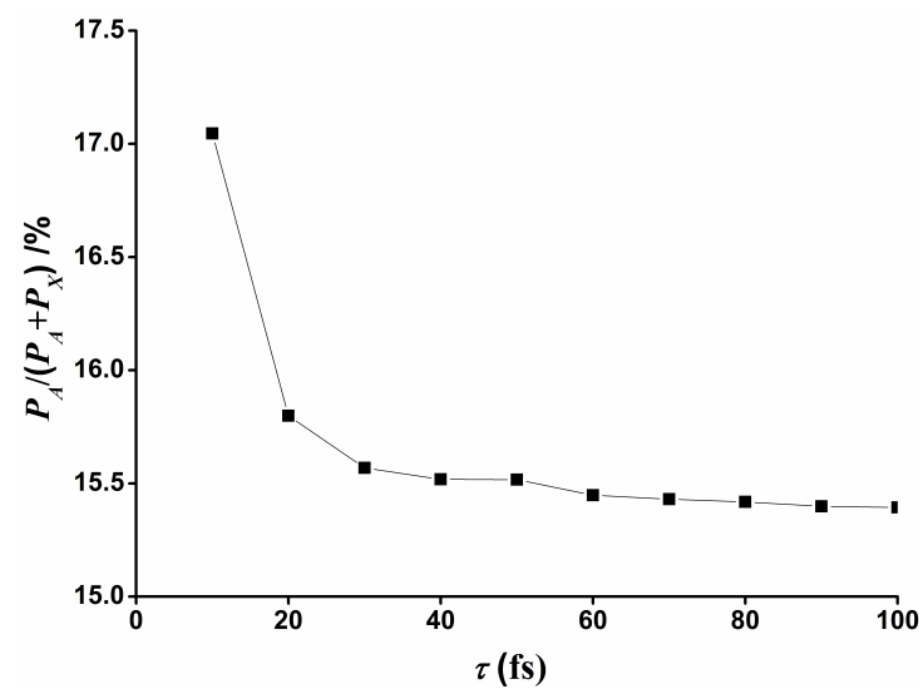

Fig. 4. The first dissociation probability vs. pulse width.

The effect of the laser wavelength on state populations is considered. Figure 5 demonstrates the evolution of state populations at different pump wavelengths (278-368 nm). After the pump laser excites the molecules to the excited state, the population on ground state achieves the minimum while that on the excited state achieves the maximum at the resonance pump wavelength $318 \mathrm{~nm}$, which indicates the maximum excitation probability. This is consistent with the studies on the wavelength dependence of state populations of $\mathrm{CsI} / \mathrm{NaLi} / \mathrm{NO}$ molecule $[13,17,18]$. Figure 6 shows the first dissociation probability $P_{A} /\left(P_{A}+P_{X}\right)$ vs. pump wavelength. The dissociation probability decreases with increasing pump wavelength. Due that the laser wavelength is inversely proportional to the laser energy, the propagation velocity of the molecule on the excited state decreases with increasing laser wavelength, which results in increasing time to reach the crossing region, leading to decreasing dissociation probability. There are few studies 
on the effect of the laser wavelength on the state populations and dissociation probability of $\mathrm{NaI}$ molecule before. Yao et al. [3] suggested that the longer the laser wavelength is (310-330 nm), the smaller the dissociation probability is. The effect of the probe wavelength $(218-258 \mathrm{~nm})$ on state populations is also studied, which shows the probe wavelength has no effect on state populations.
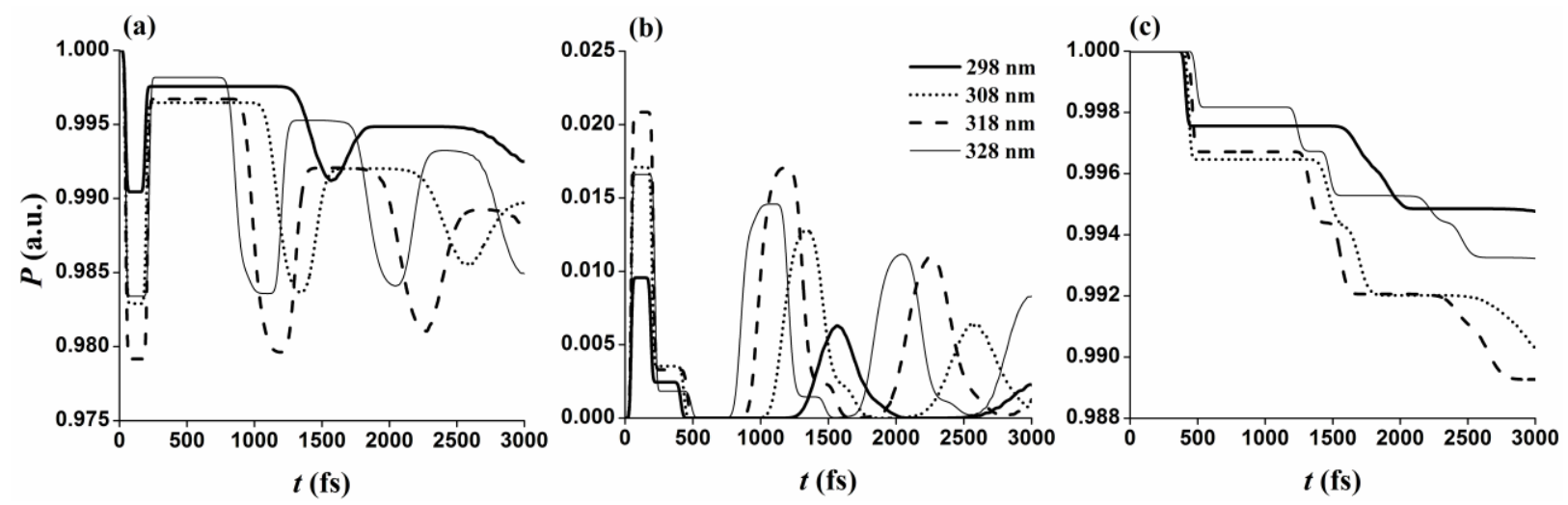

Fig. 5. The evolution of state populations at different pump wavelengths. (a), (b) and (c) denote ground state, excited state and the sum of the three states, respectively.

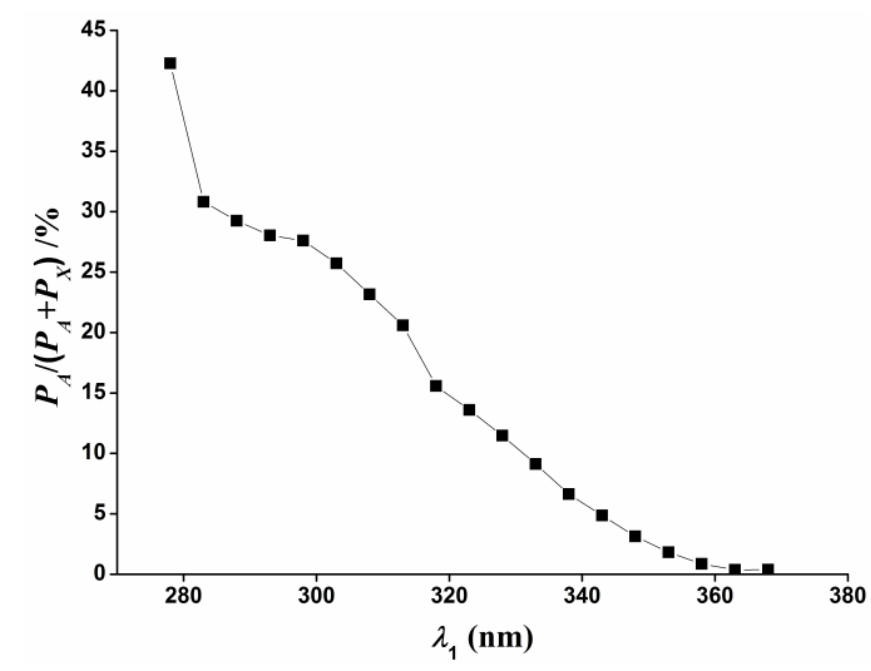

Fig. 6. The first dissociation probability vs. pump wavelength.

The effect of laser intensity on state populations is considered. Figure 7 demonstrates the evolution of state populations at different pump laser intensities. After the pump laser excites the molecules to the excited state, the decrease of the population on ground state increases while the increase of that on the excited state increases with increasing laser intensity, i.e., the excitation probability increases. The decrease of the sum of the three states increases with increasing pump laser intensities [Fig. 7(c)], but the dissociation probability does not change. The effect of the 
probe laser intensities on state populations is also studied, which shows the probe laser intensity has no effect on state populations. The effect of the laser intensity on the state populations and dissociation probability of $\mathrm{NaI}$ molecule have not studied before.
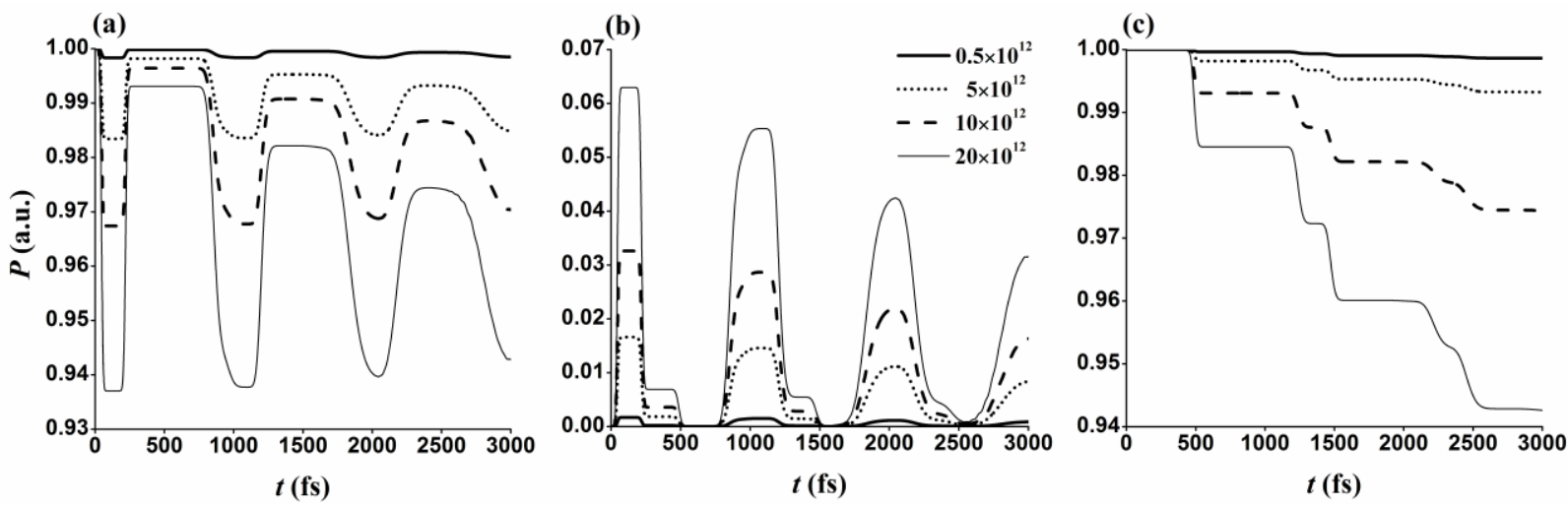

Fig. 7. The evolution of state populations at different pump intensities. (a), (b) and (c) denote ground state, excited state and the sum of the three states, respectively.

\section{Conclusion}

The effect of the pulse width, laser wavelength and intensity on state populations of $\mathrm{NaI}$ molecule driven by a pump-probe pulse were studied via employing the time-dependent wave packet approach. Wave packet moves periodically with roundtrip time $1000 \mathrm{fs}$. The wave packet reaches the crossing point firstly at 200 fs and bifurcates: most of the wave packet transfers to the ground state, only a small part still on the excited state, which affects state populations. The excitation probability increases and dissociation probability decreases with increasing pulse width. The excitation probability approaches the maximum with the resonance pump wavelength $318 \mathrm{~nm}$. The dissociation probability decreases with increasing pump wavelength. As the pump intensity increases, the excitation probability increases while the dissociation probability does not change. The probe laser wavelength and intensity do not affect the state populations. The wave packet motion, selective distribution of state populations, excitation and dissociation of molecule can be achieved by adjusting laser parameters. The results may be useful for realizing the optical control of molecule and the process of quantum manipulation of molecule experimentally, and provide some important basis for further theoretical research in this respect. 


\section{Acknowledgments}

This work was supported by the National Science Foundation of China under Grant No.11447020, the Natural Science Foundation of Hunan province under Grant No. 2015JJ3104, and the Research Foundation of Education Bureau of Hunan province under Grant No. 15B204.

\section{References}

1. M. Braun, C. Meier, V. Engel, The reflection of predissociation dynamics in pump/probe photoelectron distributions, The Journal of Chemical Physics 105 (1996) 530-534.

2. E. Charron, A. Suzor-Weiner, Femtosecond dynamics of NaI ionization and dissocitive ionization, The Journal of Chemical Physics 108 (1998) 3922-3931.

3. H.B. Yao, Y.J. Zheng, The non-adiabatic effects of NaI molecule, Acta Physica Sinica 60 (2011) 128201.

4. H.B. Yao, S.Y. Lin, Y.J. Zheng, Nonadiabatic effects in femtosecond photoionization of NaI molecule, Journal of Theoretical and Computational Chemistry 10 (2011) 509-518.

5. A. Arasaki, K. Takatsuka, K. Wang, McKoy Vincent, Studies of electron transfer in NaI with pump-probe femtosecond photoelectron spectroscopy, Journal of Chemical Physics 119 (2003) 7913-7923.

6. A. Arasaki, K. Takatsuka, K. Wang, McKoy Vincent, Pump-probe photoionization study of the passage and bifurcation of a quantum wave packet across an avoided crossing, Physical Review Letters 90 (2003) 248303.

7. K. Takatsuka, Y. Arasaki, K. Wang, McKoy Vincent, Probing wavepacket dynamics with femtosecond energy- and angle-resolved photoelectron spectroscopy, Faraday Discuss. 115 (2000) 1-15.

8. X.Y. Miao, J.F. Zhang, X.F. Jia, Probing the process of photodissociation of the NaI molecule with pump-probe femtosecond spectroscopy, EPL 82 (2008) 33001.

9. X.Y. Miao, L. Wang, H.S. Song, Theoretical study of the femtosecond photoionization of the NaI molecule, Physical Review A 75 (2007) 042512.

10. R.F. Liu, H.S. Zhai, Y.L. Gao, R.Q. Liu, Theoretical investigation of femtosecond-resolved photoelectron spectrum of NaI molecules, Chinese Physics Letters 25 (2008) 2016-2019.

11. X.G. Ma, C.L. Yang, M.S. Wang, Y.B. Gong, W.W. Liu, Femtosecond control of photoionization and photodissociation of sodium iodine molecules by laser pulse, Chinese Optics Letters 10 (2012) 110201.

12. Z.Y. Zhao, Y.C. Han, J. Yu, S.L. Cong, The influence of field-free orientation on the predissociation dynamics of the NaI molecule, The Journal of Chemical Physics 140 (2014) 044316. 
13. D.L. Xiong, M.S. Wang, C.L. Yang, X.F. Tong, N. Ma, Control of reaction channels of CsI molecule by ultra-short laser pulse, Chinese Physics B 19 (2010) 103303.

14. Y.H. Zhu, P. Song, F.C. Ma, Theoretical study of the dynamic stark effect on dissociation of CsI, Brazilian Journal of Physics 44 (2014) 189-193.

15. H. Zhang, K.L. Han, Y. Zhao, G.Z. He, N.Q. Lou, A real time dynamical calculation of $\mathrm{H}_{2}{ }^{-}$ Photodissociation, Chemical Physics Letters 271 (1997) 204-208.

16. T.S. Chu, Y. Zhang, K.L. Han, The time-dependent quantum wave packet approach to the electronically nonadiabatic processes in chemical reactions, International Reviews in Physical Chemistry 25 (2006) 201-235.

17. M. Zhang, M.S. Wang, D.L. Xiong, N. Ma, The influence of femtosecond laser parameters on the wavepacket and population of the diabatic excited states of NaLi, Molecular Physics 111 (2013) 61-71.

18. J. Wang, F. Liu, D.G. Yue, J. Zhao, Y. Xu, Q.T. Meng, Wing-Ki Liu, Influence of laser fields on the vibrational population of molecules and its wave-packet dynamical investigation, Chinese Physics B 19 (2010) 123301. 